\title{
Anti-Mullerian hormone before and after uterine artery embolisation in reproductive-age women seeking treatment for symptomatic fibroids
}

\author{
C M Muteshi, ${ }^{1}$ MB ChB, MMEd (Obsetr Gynaecol); A Murage, ${ }^{2}$ MRCOG (UK); S Ngugi, ${ }^{2}$ MMEd (Obstetr Gynaecol) \\ ${ }^{1}$ Nuffield Department of Women's and Reproductive Health, University of Oxford, UK \\ ${ }^{2}$ Department of Obstetrics and Gynaecology, Aga Khan University, Nairobi, Kenya
}

Corresponding author: C M Muteshi (murwa2006@yahoo.co.uk)

\begin{abstract}
Background. Uterine fibroids are the most common of the benign gynaecological tumours, the definitive traditional treatment for which is either myomectomy or hysterectomy. Uterine artery embolisation (UAE) offers an alternative non-invasive option for treatment. There remain concerns as to the effects of UAE on ovarian reserve in women desirous of preserving ovarian function.

Objectives. To determine the effect of UAE on ovarian reserve, as assessed by anti-Mullerian hormone (AMH) measured before and after embolisation, and to assess the trend in serum AMH in the medium term, up to 12 months post UAE.

Methods. A cohort study between October 2012 and May 2015 recruited 40 women with symptomatic uterine fibroids. Serum AMH was measured prior to embolisation and post embolisation, at 2 weeks, 6 months and 12 months. Using descriptive statistics and bivariate analysis, pre-embolisation and post-embolisation AMH concentrations were compared. $P<0.05$ was considered statistically significant.

Results. The median participant age was 38.5 years (range 31 - 45). Of the women recruited, 47.5\% (19) were nulliparous, and $72.5 \%$ ( 29 women) of the total desired a pregnancy in the future. The median (standard deviation) AMH immediately prior to embolisation was $1.3 \mathrm{ng} / \mathrm{mL}$ (1.53), and post embolisation at 2 weeks, $0.9 \mathrm{ng} / \mathrm{mL}$ (1.98); at 6 months, $2.2 \mathrm{ng} / \mathrm{mL}$ (2.63); and $12 \mathrm{months}, 3.5 \mathrm{ng} / \mathrm{mL}(1.54)(p=0.96)$. Conclusion. In the short to medium term, UAE for treatment of symptomatic fibroids was not found to be detrimental to ovarian reserve. There is a need for longer-term studies evaluating its effects on fecundity, considering that most women in the present study had a strong desire to maintain their fertility.
\end{abstract}

S Afr J Obstet Gynaecol 2018;24(2):57-60. DOI:10.7196/SAJOG.2018.v24i2.1331

Uterine fibroids are the most common benign tumours of the female genital tract. Their true prevalence is probably underestimated, as the incidence at histology is more than double the clinical incidence. ${ }^{[1]}$ Longitudinal studies have estimated that the lifetime risk of fibroids in a woman over the age of 45 years is $>60 \%$, with the incidence higher in black women than in white. The fibroids may be asymptomatic, or may cause symptoms such as abnormal uterine bleeding, urinary incontinence, a feeling of pelvic pressure or pain. They may also be associated with reproductive problems such as infertility and miscarriage..$^{[1]}$

Treatment for symptomatic fibroids includes medical and surgical options such as hysterectomy and myomectomy. More recently, uterine artery embolisation (UAE) has been incorporated as one of the management options for symptomatic uterine fibroids. ${ }^{[2]}$ However, concerns remain as to the effects of embolisation on ovarian reserve, and hence fertility. ${ }^{[3]}$ Studies have been conducted to indirectly assess the ovarian reserve. A 2004 prospective study assessing 20 reproductive-age women following UAE found no significant changes from baseline in the mean day- 3 folliclestimulating hormone (FSH) and oestradiol levels, ovarian volume measurements, and antral follicle numbers measured at 3,6 and 12 months after UAE. ${ }^{[4]}$

Assessing ovarian reserve with FSH is problematic, and antiMullerian hormone (AMH) has proved to be more reliable..$^{[5]}$ Hehenkamp et al ${ }^{[6]}$ reported that incorporating AMH in protocols assessing ovarian reserve showed compromise to ovarian function following UAE. Their study, however, included older women without reproductive ambition.

Our study aimed to establish the effect of UAE on AMH measured immediately before, and 2 weeks after, embolisation. A secondary outcome was to determine the trend in $\mathrm{AMH}$ concentration at 6 and 12 months following embolisation.

\section{Methods}

We undertook a cohort study in women with symptomatic fibroids who opted for conservative fibroid treatment with UAE between October 2012 and May 2015, at Aga Khan University Hospital, Nairobi. The study was approved by the hospital research ethics committee (ref. no. 112014EA).

\section{Selection of cases}

Women attending the gynaecology outpatient clinic at Aga Khan University Hospital, Nairobi, and having a diagnosis of symptomatic uterine fibroids, were given written information about UAE. Those opting for UAE were invited to participate in the study on the day of admission for the procedure. Further written information about the study was provided, and those aged $<45$ years who desired a future pregnancy, regardless of their reproductive history, were recruited, following written informed consent. Women were excluded if they had a history of ovarian cystectomy, ovarian endometrioma or tubal 
sterilisation, or had no desire for future fertility, or were not willing to return for follow-up blood tests.

\section{Study protocol}

The protocol and procedures for UAE at the Aga Khan University Hospital are reported elsewhere. ${ }^{[7]}$ Blood was collected in plain vacutainers, and delivered to the biochemistry laboratory within 60 minutes. This was centrifuged, and sera frozen at $-20^{\circ} \mathrm{C}$ until analysis using the Beckman Coulter Gen. II assay (Beckman Coulter Inc., USA). Samples were collected immediately prior to UAE, and at 2 weeks, 6 months and 12 months following UAE.

\section{Outcome measures}

The primary outcome was change in the median concentration of serum AMH from baseline to 2 weeks following UAE. The secondary outcome was the trend in AMH concentration up to 12 months following UAE.

\section{Sample size calculation and statistical analysis}

A power calculation determined that a sample size of 65 women was needed to demonstrate a $10 \%$ difference in the before-andafter $\mathrm{AMH}$ concentration, with $80 \%$ power and a $5 \%$ significance threshold. Using univariate and bivariate analysis, data were analysed and presented as proportions, median and standard deviation. Student's $t$-test was used to compare differences, $p<0.05$ was considered significant. All statistical analysis was performed using the statistical software SPSS version 24.0 (IBM., USA).

\section{Results}

Forty women with symptomatic uterine fibroids undergoing UAE were recruited to participate in the study. Their median age at recruitment was 38.5 years (range 31 - 45 ) (Table 1).

Immediately prior to UAE, the baseline median (standard deviation (SD) serum AMH was $1.3 \mathrm{ng} / \mathrm{mL}$ (1.53). The serum AMH showed progressive decline with woman's increasing age at baseline (Pearson's correlation coefficient $r^{2}=-0.417$ ). Measured 2 weeks after the UAE, the correlation coefficient between AMH and age was $r^{2}=-0.52$, which was not significantly different to that prior to UAE $(p=0.622)$ (Fig. 1). When the median concentration of serum AMH prior to UAE was compared with the concentration immediately after embolisation, there was no statistically significant difference ( $p=0.99$ ), with the trend showing an initial slight dip, followed by a recovery at 6 months, and no return to the former levels at 12 months (Fig. 2).

\section{Discussion}

Assessment of ovarian reserve in reproductive-age women is usually performed using various static and dynamic biomarkers,

\begin{tabular}{ll} 
Table 1. Participant characteristics $(N=\mathbf{4 0})$ \\
\hline Characteristic & Value \\
\hline $\begin{array}{l}\text { Age, mean (range) } \\
\text { Parity, } n \text { (\%) }\end{array}$ & $38.5(31-45)$ \\
0 & $19(47.5)$ \\
$\quad \geq 1$ & $21(52.5)$ \\
Fertility desired, $n(\%)$ & \\
$\quad$ Yes & $29(72.5)$ \\
No & $11(27.5)$
\end{tabular}

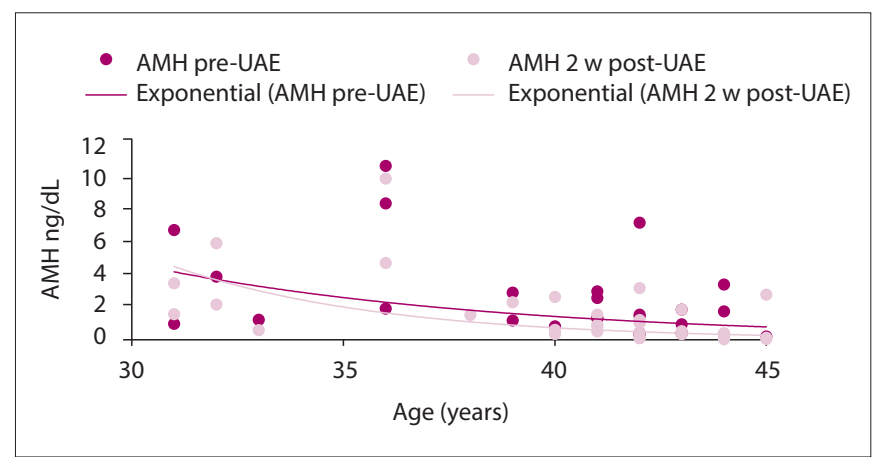

Fig. 1. AMH changes with age before and 2 weeks after UAE. Pearson's correlation coefficient before $\left(r^{2}=-0.41\right)$ and 2 weeks after $\left(r^{2}=-0.52\right)$ UAE; $\mathrm{p}=0.62$. (AMH $=$ anti-Mullerian hormone; $U A E=$ uterine artery embolisation.)

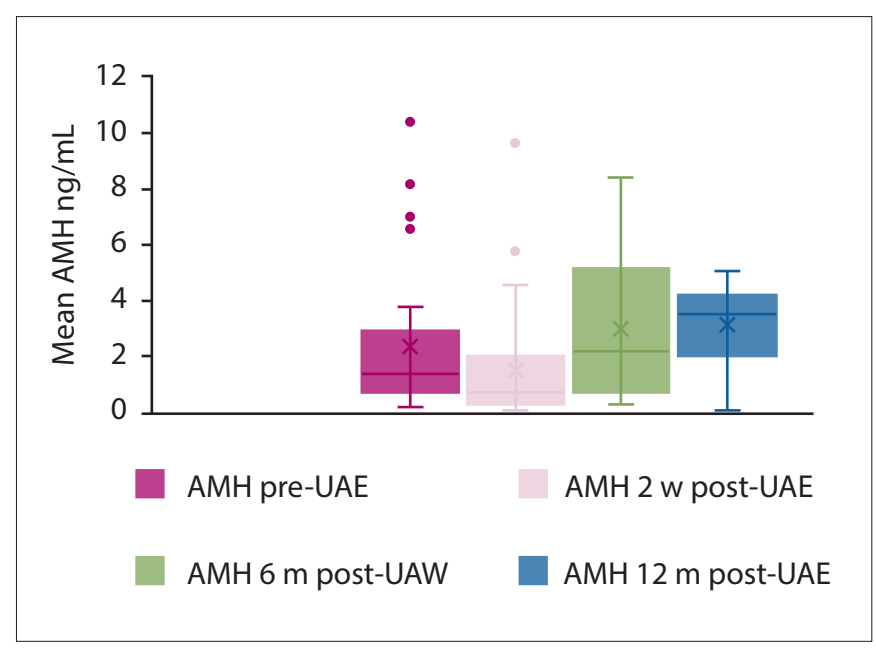

Fig 2. Trend in median AMH concentration before and after UAE, at 2 weeks, 6 months and 12 months $(\mathrm{p}=0.94) .(A M H=$ anti-Mullerian hormone; UAE = uterine artery embolisation.)

with various degrees of diagnostic accuracy. Serum FSH, oestradiol and inhibin B are less specific, cycle-dependent and are not able to reliably differentiate women with poor ovarian reserve from those with normal ovarian reserve. ${ }^{[8]}$ Transvaginal ultrasound scanning for estimating ovarian antral follicle count is reliable, cycle-independent and performs well in the differentiation of lowovarian-reserve from normal-ovarian-reserve women. Ultrasound scanning, however, is dependent on probe resolution, and requires trained operators and easy access to the ovary through the transvaginal approach. ${ }^{[9]}$ In women with large or multiple uterine fibroids, assessment of antral follicle count may be suboptimal. $\mathrm{AMH}$ is a serum biomarker for ovarian reserve testing that is reliable, cycle-independent and stable in various laboratory conditions. ${ }^{[5]}$ Our study assessed AMH values before and after UAE, showing that serum AMH levels consistently decreased immediately following UAE procedures, as early as 2 weeks later. Interestingly, AMH values had recovered on further follow-up at 6 months, and had not returned to the former levels 12 months later, showing that the initial depression is only temporary.

Traditionally, uterine fibroids are managed surgically, either by hysterectomy or, for women concerned about future fertility, by myomectomy. Hysterectomy is a major operation that carries significant risks and a variable period of convalescence. ${ }^{[10]}$ In 
the last two decades, UAE became available for women with symptomatic fibroids who wish to avoid hysterectomy, with several case reports confirming its effectiveness. ${ }^{[2,6,11,12]}$ Whereas the results of the procedure were clinically satisfactory compared with those of hysterectomy, it became apparent that for women aged $>45$ years, compromised ovarian reserve was observed in both the UAE and hysterectomy groups. This observation raised questions about the safety of UAE in terms of ovarian function. ${ }^{[6]}$

The effect of UAE on ovarian reserve in younger women has been inconsistent across studies, and therefore remains uncertain, prompting the recommendation that for women desiring future pregnancy, caution should be observed when considering UAE. ${ }^{[13-16]}$ Our findings are comparable with those reported in studies of younger women who had UAE and ovarian reserve assessed more accurately with serum $\mathrm{AMH}$ before and after the procedure. Of note is an initial dip in AMH concentration in the period immediately after the procedure, followed by recovery before a steady natural decline. ${ }^{[6,15]}$ The mechanism by which UAE affects ovarian reserve is not completely understood, and explanations remain speculative. It is hypothesised that non-target embolisation causes hypoxia to the ovarian cortex, resulting in loss of AMH-secreting small antral and pre-antral follicles that are sensitive to reduced tissue oxygen tension. ${ }^{[17]}$ This is likely the case in women with utero-ovarian anastomosis, since embolic material has been recovered from ovarian biopsy following UAE. ${ }^{[18]}$ However, this may be transient, as recovery of antral follicles from the primordial follicle pool happens with time. ${ }^{[6,18]}$ The reports of permanent ovarian failure in older women would suggest already severely compromised ovarian reserve, from which the transient loss is too great to make a full recovery.

No studies have explicitly determined the critical ovarian reserve that predicts complete ovarian failure following UAE; however, studies that evaluate ovarian failure following gonadotoxic treatment show that the risk of ovarian failure is dependent on ovarian reserve and age. ${ }^{[19]}$ Clearly, UAE off-target damage to the ovary is unlikely to be similar in magnitude to damage caused by chemotherapy or radiation therapy. The Wallace-Kelsey model ${ }^{[20]}$ elegantly demonstrates that in $95 \%$ of women, by the age of 30 , only $12 \%$ of the maximum pre-birth non-growing follicle population is present, decreasing further to $3 \%$ by the age of 40 . It is therefore likely that by the age of 45 , when clear evidence of damage from embolisation or hysterectomy is seen, the non-growing follicle pool is even lower, as shown in studies reporting permanent ovarian failure. ${ }^{[6,14]}$ It is well known that reproductive competence decreases with maternal age, mainly owing to reduced ovarian reserve. ${ }^{[2]}$ Accurate evaluation of ovarian reserve using serum AMH level shows that values vary greatly across a population of women of the same age; therefore, women may experience premature ovarian insufficiency while still having regular periods and normal gonadotropins. ${ }^{[2-24]}$

In our study, the median (SD) female age was 38.5 (6.5) years, with a median serum AMH of 1.3 (1.53) ng/mL before embolisation, and future fertility aspirations in up to $72.5 \%$ of the participants. It is not surprising that nearly three-quarters of the women still had fertility aspirations, as is comparable with other studies that report that many of those considering options for fibroid management usually opt for fertility preservation or conservative procedures. ${ }^{[25]}$ The effect of fibroids on infertility is, however, less clear, and may be indistinguishable from age-related effects on fecundity, considering that the incidence of fibroids increases with age, with studies reporting varying findings. ${ }^{[26,27]}$
Generally, the chance of natural conception is inversely proportional to female age, with a proportionate increase in the risk of miscarriage. ${ }^{[28,29]}$ Women being investigated for subfertility and found to have fibroids must consider this very carefully, particularly when choosing which treatment to pursue. Whereas the cohort of women in our study only indicated a desire for future pregnancy, information on those who had had previous fertility investigations was not sought. Reassuringly, the mean AMH levels prior to embolisation were within the expected normal range for age. It would therefore be expected that women aged $<40$ years would have an $80 \%$ chance of conceiving a pregnancy after trying for 12 months with no other cause for delay identified. ${ }^{[30]}$ UAE did not result in decreased concentration of AMH in the short term, but we were unable to determine reproductive outcome owing to a lack of follow-up information. Considering that ovarian failure following UAE is reported more commonly in women aged $\geq 45$ years, and in younger women whose AMH concentration falls below the fifth percentile, consideration should be given to options for fibroid treatment other than embolisation, as these women have the highest risk of ovarian failure. ${ }^{[23]}$ In addition, concerns have also been raised about increased risks of pregnancy complications in women who have had UAE. ${ }^{[2]}$

Our study benefitted from measuring serum AMH before and after UAE at fixed time points. AMH is a robust biomarker and also independent of the ovarian cycle, with high predictability of both ovarian reserve and reproductive ageing. ${ }^{[5]}$ The inclusion of women up to the age of 45 years in this trial allows for generalisability of the findings, as changing sociodemographic characteristics of reproductive-age women embarking on their first pregnancy in their late 30 s or early 40 s become a reality. ${ }^{[31]}$

Our study was limited by recruiting fewer women than initially anticipated. This renders the results subject to cautious interpretation; however, the general trend observed in $\mathrm{AMH}$ values conforms with that found in other trials, and is likely to represent a true biological phenomenon after embolisation. ${ }^{[15]}$ In trials using other markers of ovarian function, a similar observation was reported. ${ }^{[14]}$ Whereas a single-point AMH measurement of ovarian reserve is predictive of reproductive function, it is essential to design trials with longer-term follow-up to establish whether the trajectory in $\mathrm{AMH}$ decline is maintained at the same rate, or whether it accelerates beyond the first year. This will be especially informative for younger women with significant fibroid symptoms and normal ovarian reserve, who wish to avoid an operative procedure such as myomectomy. It is likely that younger women whose AMH values fall below the fifth percentile will have the highest risk of ovarian failure following UAE; however, a study to test this hypothesis would be ethically unsound, and conclusions can only be made from logical reasoning.

\section{Conclusion}

From this study, we can conclude that in women with symptomatic uterine fibroids and normal ovarian reserve estimated using $\mathrm{AMH}$, UAE does not compromise ovarian function in the shorter to medium term. For women with fibroids seeking treatment who have future fertility aspirations, part of the investigations should include ovarian reserve testing, to ensure that they receive adequate and complete information to make an informed decision on the most appropriate management. It will be interesting to see whether the neutral effect of UAE on ovarian reserve is maintained in the longer term, and what its impact might be on female reproductive biology. 


\section{RESEARCH}

\section{Acknowledgements. None.}

Author contributions. CM developed the study protocol, applied for funding, analysed data and wrote the initial draft manuscript. AM developed the draft protocol, applied for funding, collected data and reviewed the draft manuscript. SN collected data and reviewed the draft manuscript. All authors approved the final manuscript for submission.

\section{Conflicts of interest. None.}

Funding. This study was funded by a grant from Aga Khan University Research Council, Project ID: 112014EA.

1. Okolo S. Incidence, aetiology and epidemiology of uterine fibroids. Best Pract Res Clin Obstet Gynaecol 2008;22(4):571-588. https://doi.org/10.1016/j.bpobgyn.2008.04.002

2. Gupta JK, Sinha A, Lumsden MA, Hickey M. Uterine artery embolisation for symptomatic uterine fibroids. Cochrane Database Syst Rev 2014(12):Cd005073. https://doi.org/10.1002/14651858. CD005073.pub4

3. Tulandi T, Salamah K. Fertility and uterine artery embolisation. Obstet Gynecol 2010;115(4):857 860. https://doi.org/10.1097/AOG.0b013e3181d4891e

4. Tropeano G, Di Stasi C, Litwicka K, Romano D, Draisci G, Mancuso S. Uterine artery embolisation for fibroids does not have adverse effects on ovarian reserve in regularly cycling women younge than 40 years. Fertil Steril 2004;81(4):1055-1061. https://doi.org/10.1016/j.fertnstert.2003.09.046

5. Broer SL, Broekmans FJ, Laven JS, Fauser BC. Anti-Mullerian hormone: Ovarian reserve testing and its potential clinical implications. Hum Reprod Update 2014;20(5):688-701. https://doi.org/10.1093/ humupd/dmu020

6. Hehenkamp WJK, Volkers NA, Broekmans FJM, et al. Loss of ovarian reserve after uterine arter embolisation: A randomised comparison with hysterectomy. Hum Reprod 2007;22(7):1996-2005. https://doi.org/10.1093/humrep/dem105

7. Mutai J, Vinayak S, Stones W, Hacking N, Mariara C. Uterine fibroid embolisation for symptomatic fibroids: Study at a teaching hospital in Kenya. J Clin Imaging Sci 2015;5:18. https://doi. fibroids: Study at a teaching
org/10.4103/2156-7514.154351

org/10.4103/2156-7514.154351 8. La Marca A, Broekmans Fo we know? Hum Reprod 2009;24(9):2264-2275. https://doi.org/10.1093/ humrep/dep210

9. Jayaprakasan K, Hilwah N, Kendall NR, et al. Does 3D ultrasound offer any advantage in the pretreatment assessment of ovarian reserve and prediction of outcome after assisted reproduction pretreatment assessment of ovarian reserve and prediction of outcome after assisted rep
treatment? Hum Reprod 2007;22(7):1932-1941. https://doi.org/10.1093/humrep/dem104

10. Garry R, Fountain J, Mason S, et al. The eVALuate study: Two parallel randomised trials, on comparing laparoscopic with abdominal hysterectomy, the other comparing laparoscopic wit vaginal hysterectomy. BMJ 2004;328(7432):129. https://doi.org/10.1136/bmj.37984.623889.F6

11. Marshburn PB, Matthews ML, Hurst BS. Uterine artery embolisation as a treatment option for uterine myomas. Obstet Gynecol Clin North Am 2006;33(1):125-144. https://doi.org/10.1016/j. ogc.2005.12.009

12. Dutton S, Hirst A, McPherson K, Nicholson T, Maresh M. A UK multicentre retrospective cohor study comparing hysterectomy and uterine artery embolisation for the treatment of symptomatic uterine fibroids (HOPEFUL study): Main results on medium-term safety and efficacy. BJOG 2007;114(11):1340-1351. https://doi.org/10.1111/j.1471-0528.2007.01526.
13. Tulandi T, Sammour A, Valenti D, Child TJ, Seti L, Tan SL. Ovarian reserve after uterine artery embolisation for leiomyomata. Fertil Steril 2002;78(1):197-198.

14. Rashid S, Khaund A, Murray LS, et al. The effects of uterine artery embolisation and surgical treatment on ovarian function in women with uterine fibroids. BJOG 2010;117(8):985-989. https:// doi.org/10.1111/j.1471-0528.2010.02579.x

15. McLucas B, Voorhees WD, Chua KJC. Anti Mullerian hormone levels before and after uterine artery embolisation: A preliminary report. Minim Invasive Ther Allied Technol 2015;24(4):242-245. https://doi.org/10.3109/13645706.2015.1012084

16. Czuczwar P, Stepniak A, Wrona W, Wozniak S, Milart P, Paszkowski T. The influence of uterine artery embolisation on ovarian reserve, fertility, and pregnancy outcomes - a review of literature. Prz Menopauzalny 2016;15(4):205-209. https://doi.org/10.5114/pm.2016.65665

17. Hascalik S, Celik O, Sarac K, Hascalik M. Transient ovarian failure: A rare complication of uterine fibroid embolisation. Acta Obstet Gynecol Scand 2004;83(7):682-685. https://doi.org/10.1111/ j.0001-6349.2004.0226a.x

18. Payne JF, Robboy SJ, Haney AF. Embolic microspheres within ovarian arterial vasculature after uterine artery embolisation. Obstet Gynecol 2002;100(5 Pt 1):883-886.

19. Meirow D, Biederman H, Anderson RA, Wallace WH. Toxicity of chemotherapy and radiation on female reproduction. Clin Obstet Gynecol 2010;53(4):727-739. https://doi.org/10.1097/ GRF.0b013e3181f96b54

20. Wallace WH, Kelsey TW. Human ovarian reserve from conception to the menopause. PLoS One 2010;5(1):e8772. https://doi.org/10.1371/journal.pone.0008772

21. Macklon NS, Fauser BC. Ovarian reserve. Semin Reprod Med 2005;23(3):248-256. https://doi. org $/ 10.1055 / \mathrm{s}-2005-872453$

22. De Vet A, Laven JS, de Jong FH, Themmen AP, Fauser BC. Anti-Mullerian hormone serum levels: A putative marker for ovarian aging. Fertil Steril 2002;77(2):357-362.

23. Van Rooij IA, Tonkelaar I, Broekmans FJ, et al. Anti-Mullerian hormone is a promising predictor fo the occurrence of the menopausal transition. Menopause 2004;11(6 Pt 1):601-606.

24. De Kat AC, van der Schouw YT, Eijkemans MJ, et al. Back to the basics of ovarian aging: A population-based study on longitudinal anti-Mullerian hormone decline. BMC Med 2016;14(1):15 https://doi.org/10.1186/s12916-016-0699-y

25. Borah BJ, Nicholson WK, Bradley L, Stewart EA. The impact of uterine leiomyomas: A nationa survey of affected women. Am J Obstet Gynecol 2013;209(4):319.el-e20. https://doi.org/10.1016/j. ajog.2013.07.017

26. Kroon B, Johnson N, Chapman M, Yazdani A, Hart R. Fibroids in infertility - consensus statement from ACCEPT (Australasian CREI Consensus Expert Panel on Trial evidence). Aust N Z J Obstet Gynaecol 2011;51(4):289-295. https://doi.org/10.1111/j.1479-828X.2011.01300.x

27. Baird DD, Dunson DB, Hill MC, Cousins D, Schectman JM. High cumulative incidence of uterine leiomyoma in black and white women : ultrasound evidence. Am J Obstet Gynecol 2003:188(1):100-107.

28. Heffner LJ. Advanced maternal age - how old is too old? N Engl J Med 2004;351(19):1927-1929.

29. Nybo Andersen AM, Wohlfahrt J, Christens P, Olsen J, Melbye M. Maternal age and fetal loss: Population-based register linkage study. BMJ 2000;320(7251):1708-1712.

30. Dunson DB, Baird DD, Colombo B. Increased infertility with age in men and women. Obstet Gynecol 2004:103(1):51-56. https://doi.org/10.1097/01_AOG.0000100153.24061.45

31. Mills M, Rindfuss RR, McDonald P, te Velde E. Why do people postpone parenthood? Reason and social policy incentives. Hum Reprod Update 2011;17(6):848-860. https://doi.org/10.1093/ humupd/dmr026

Accepted 27 October 2018 